\title{
A study of oligoclonal band negative multiple sclerosis
}

\author{
A Z J Zeman, D Kidd, B N McLean, M A Kelly, D A Francis, D H Miller, B E Kendall, \\ P Rudge, E J Thompson, W I McDonald
}

\begin{abstract}
Objectives-to determine whether oligoclonal band (OCB) negative multiple sclerosis is a reliable diagnosis and, if so, whether it has a distinctive prognosis.

Methods-retrospective and matched prospective comparison of the clinical and laboratory features of patients with clinical definite multiple sclerosis with and without intrathecal synthesis of oligoclonal IgG.

Results-thirty four patients were identified with apparent OCB negative clinically definite multiple sclerosis. The results of oligoclonal banding proved to have been equivocal in 14 of 34 ; the clinical diagnosis of multiple sclerosis was questionable in 8 of 34 . The remaining 12 patients with "true" OCB negative multiple sclerosis were significantly less disabled than matched OCB positive controls. Re-examination of CSF-serum pairs from six OCB negative patients showed that three remained OCB negative while three showed evidence of intrathecal synthesis of OCBs.

Conclusions-OCB negative clinically definite multiple sclerosis is rare and should be diagnosed with caution; in unequivocal cases it seems to have a relatively benign prognosis.
\end{abstract}

\section{(F Neurol Neurosurg Psychiatry 1996;60:27-30)}

Keywords: multiple sclerosis; oligoclonal bands

Institute of Neurology, University of London, Queen Square, London WC1N 3BG, UK

A Z J Zeman

$D$ Kidd

B N McLean

D H Miller

B E Kendall

P Rudge

E J Thompson

W I McDonald

Department of

Neurology, University

of Birmingham, Queen

of Birmingham, Qu

Birmingham B15 2TH,

UK

M A Kelly

D A Francis

Correspondence to:

Dr Adam Zeman

Department of Neurology,

Aduls nood sospital

CB2 $2 Q Q$ UK.

Received 24 February 1995

and in final revised form

14 September 1995

Accepted 21 September 1995 represent a first attack of multiple sclerosis, such as optic neuritis or transverse myelitis, evidence of intrathecal synthesis of oligoclonal IgG or IgM indicates an increased likelihood of progression to clinically definite disease. ${ }^{7-9}$ The contribution of intrathecal IgG synthesis to the pathogenesis of multiple sclerosis, and its bearing on the aetiology of the disease, remain uncertain. ${ }^{310}$

Occasional patients with clinically definite multiple sclerosis lack evidence for intrathecal IgG synthesis. Rudick et al identified the absence of such evidence as one of five "red flag" features which should cast doubt on the diagnosis. ${ }^{11}$ However, the postmortem study of Farrell et al confirmed that OCBs may be absent in multiple sclerosis established by necropsy. ${ }^{12}$ Their absence was associated with low numbers of "active plaques" and of plasma cells in affected and unaffected white matter and meninges. Stendahl-Brodin et al concluded from their clinical comparison of OCB negative and positive patients that the absence of OCBs predicts a relatively benign prognosis. ${ }^{13}$

This study was designed to re-examine the practical and theoretical questions raised by OCB negative critically definite multiple sclerosis with the aid of up to date techniques of immunochemistry and neuroimaging.

\section{Methods}

PATIENTS AND STUDY DESIGN

The case notes of patients admitted to The National Hospital for Neurology and Neurosurgery for investigation of suspected multiple sclerosis between 1985 and 1990 were reviewed. Patients with clinically definite or progressive probable disease ${ }^{14}$ whose CSF and serum had been examined by isoelectric focusing were identified. Some of these patients were OCB negative. A few additional patients were identified after a request to clinicians at the National Hospital to report any cases of OCB negative definite multiple sclerosis encountered in their clinics.

The clinical details and stored laboratory results of patients with putative OCB negative multiple sclerosis were scrutinised to identify patients with plausible alternative diagnoses to multiple sclerosis (WIM), and those with questionable laboratory results (EJT).

We compared the OCB negative and positive patients in two ways:

(1) the clinical features of the two groups, ascertained retrospectively from the notes, were compared.

(2) We performed a prospective comparison of the OCB negative patients with matched OCB positive controls. Each patient in whom both 
the clinical and the laboratory diagnoses seemed secure was matched with an OCB positive patient drawn from the group of patients investigated in 1989-90 for sex, age, duration of disease, Poser category, and disease type (matching of the relapsing and remitting to the secondary progressive type was permitted). ${ }^{15}$

Matching was performed in the following manner. For each OCB negative case we identified the OCB positive patients investigated in 1989-90 who were of the same sex, within five years in age and duration of disease, and who were of the same diagnostic category and type. If one of the potential controls was closer to the index case in both age and duration of disease than any other, that control was selected for further study. If not, random numbers were assigned to the potential controls, and the control with the lowest random number was selected. The matching constraints were relaxed in two cases: an $O C B$ negative patient with an exceptionally long duration of disease (37 years) was matched with an OCB positive control with a disease duration of 22 years; a 58 year old OCB negative patient was matched with an OCP positive control aged 50.

Consenting matched OCB negative and OCB positive patients underwent: $(a)$ a full history and neurological examination (AZJZ); (b) a blind disability assessment, using the Kurtzke expanded disability status scale

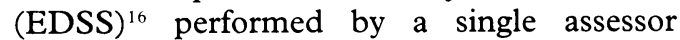
(DK); (c) MRI of brain and spinal cord before and after an infusion of gadolinium-DTPA; (d) venepuncture for HLA typing and immunochemistry (all patients); (e) lumbar puncture for CSF immunochemistry (oligoclonal band negative patients only). Patients who consented to $(a)$ and $(b)$ only were nevertheless included in the study.

\section{IMMUNOCHEMISTRY}

Both initial and follow up analysis of CSFserum pairs involved: (a) isoelectric focusing of CSF and serum with protein transfer to nitrocellulose and visualisation of IgG using an enzyme labelled double antibody method as previously described ${ }^{17}$; (b) polyacrylamide gel electrophoresis (PAGE) of $\mathrm{CSF}^{18}$; (c) immunoblotting of the PAGE gel with visualisation of free and bound light chains. ${ }^{18}$

\section{MAGNETIC RESONANCE IMAGING}

Brain and spinal cord MRI was performed in nine consenting OCB negative-OCB positive patient pairs with a Signa $1.5 \mathrm{~T}$ superconducting system (GE Milwaukee), before and after enhancement with $0.01 \mathrm{mmol} / \mathrm{kg}$ gadolinium DTPA (two patients declined enhancement). Axial $\mathrm{T} 2$ and proton density weighted images of the brain were acquired using a fast spin echo (FSE) sequence (13 patients) or a standard dual spin echo sequence (five patients). Sagittal FSE images of the spinal cord were obtained in 13 patients, standard spin echo images in five. Axial images of the cord were acquired using a gradient echo sequence.

Scans were reported by a neuroradiologist (BEK) who was blinded to clinical details.
Lesions in brain and spinal cord were quantified as previously described. ${ }^{1920}$

\section{HLA TYPING}

Genomic DNA was isolated from $30 \mathrm{ml}$ venous blood by a phenol-chloroform extraction procedure. DR, DQA1, and DQB1 alleles were determined by standard methods.

\section{STATISTICS}

Statistical analysis was performed using the Arcus statistical package. The Wilcoxon signed rank test for non-parametric data was used to compare the characteristics of the matched OCB negative and OCB positive patient pairs. The Mann-Whitney test and the $\chi^{2}$ squared test with Yates' correction were used to compare the characteristics of the OCB positive and negative patient groups.

\section{Results}

PREVALENCE OF OCB NEGATIVE CLINICALLY DEFINITE MULTIPLE SCLEROSIS

Two hundred and ninety patients with clinically definite or progressive probable multiple sclerosis had their CSF examined by isoelectric focusing at the National Hospital between 1985 and 1990. Thirty one were OCB negative, 25 of whom were clinically definite, six progressive probable. ${ }^{14}$ Three further OCB negative patients, all clinically definite, were identified by clinicians.

On review, the original laboratory results proved to be equivocal in 13 patients and in error in one. The reasons for equivocal results were suboptimal loading of CSF IgG at isoelectric focusing in four cases and the presence of extremely faint bands which had not been reported in nine cases.

Review of the clinical records disclosed the possibility of an alternative diagnosis to multiple sclerosis in five cases, and two cases were so atypical of multiple sclerosis that it was considered unwise to include them. The alternative diagnoses were: inherited cerebellar degeneration in two cases, and systemic lupus erythematosus, idiopathic cortical degeneration, and hysteria in one case each.

Thirteen cases remained in which both clinical diagnosis and laboratory result were considered secure. All were clinically definite by the criteria of Poser $^{6}$ and either relapsing and remitting or secondary progressive in type. One of these "true" OCB negative patients proved to have cervical cord compression as an alternative, or additional, diagnosis to multiple sclerosis on the basis of investigations performed as a part of this study. Thus the final OCB negative group contained 12 patients (table 1). Nine derived from the group of patients studied between 1985 and 1990 , indicating a frequency of true clinically definite OCB negative multiple sclerosis of $3 \cdot 1 \%$ (nine of 290 ) in this population.

\section{COMPARISON OF OCB NEGATIVE WITH OCB} POSITIVE PATIENTS

\section{Retrospective group comparison}

Table 1 shows the comparison of the clinical and laboratory features of the OCB negative 
Table 1 Comparison of clinical and laboratory features of $O C B$ negative patients and $O C B$ positive control patients 1989-90

\begin{tabular}{lccl}
\hline & OCB positive & OCB negative & P value \\
\hline Sex ratio (F:M) & $94: 50(1 \cdot 9: 1)$ & $6: 6(1: 1)$ & NS \\
Poser (CDMSA 1:2) & $105: 39(2 \cdot 7: 1)$ & $8: 4(2: 1)$ & NS \\
Type (RR:2 $\left.2^{\circ}\right)$ & $94: 50(1 \cdot 9: 1)$ & $7: 5(1 \cdot 4: 1)$ & NS \\
Mean age (y) & $41(41)$ & $42(43)$ & NS \\
Mean duration & $11(10)$ & $14(12)$ & NS \\
Mean duration to LP & $8(6)$ & $11(10)$ & $0 \cdot 02$ \\
Disability & $4(4)$ & $3(3 \cdot 5)$ & $0 \cdot 0005$ \\
Polyacryl OCBs & $82 / 128$ & $0 / 12$ & $<0 \cdot 0001$ \\
Total light chains & $95 / 115$ & $0 / 12$ & 0.0001 \\
Free light chains & $72 / 115$ & $0 / 12$ & \\
& & 12 & \\
Total & 144 & 12 &
\end{tabular}

Poser $\left(\right.$ CDMSA1:CDMSA2) = ratio of patients with CDMSA1 by the Poser classification ${ }^{5}$ patients with CDMSA2; type $\left(R R: 2^{\circ} \mathrm{P}\right)=$ ratio of patients with relapsing and remitting to patients with secondary progressive disease ${ }^{14}$; mean duration $=$ mean duration of disease from onset of symptoms; mean duration to LP = mean interval between onset of symptoms and initia lumbar puncture; disability $=$ mean Kurtzke EDSS, ${ }^{15}$ assessed retrospectively from the clinica notes; polyacryl OCBs = numbers of patients with oligoclonal bands on polyacrylamide gel electrophoresis ${ }^{17}$; total light chains = numbers of patients in whom CSF light chains were detected by immunobloting of polyacrylamide gels $^{17}$; free light chains $=$ numbers of patients in whom free light chains were detected by immunoblotting of polyacrylamide gels.

group with the group of clinically definite OCB positive patients admitted in 1989-90.

Prospective case-control comparison

Eleven of 12 OCB negative patients consented to further study. Eleven OCB positive patients were matched for age, sex, duration of disease, Poser category, and disease type. Ten of these patients agreed to take part; one patient failed to respond to two approaches. Another suitable OCB positive patient was approached and agreed to participate.

There were no significant differences in age or duration of disease between patients in the OCB negative-OCB positive pairs by the Wilcoxon signed rank test. Sex and Poser group (clinically definite multiple sclerosis) were identical within each pair. Six OCB negative patients were relapsing and remitting, five secondary progressive; three OCB positive patients were relapsing and remitting, eight secondary progressive.

Disability was assessed in every patient. Table 2 summarises the scores.

Brain and spinal cord MRI was performed in all $11 \mathrm{OCB}$ positive patients with gadolinium enhancement in 10, and in nine OCB negative patients with gadolinium enhancement in eight. The spinal cord images were technically unsatisfactory in one OCB negative patient.

Table 2 summarises the cranial and lesion loads. Although the cranial lesion score was higher in the OCB positive patients in seven of nine pairs these differences were not significant at $\mathrm{P}<0.05$ (Wilcoxon signed rank test one tailed $P=0 \cdot 21$ ). Gadolinium enhancing lesions on cranial MRI were infrequent in both

Table 2 Kurtzke disability scores and MRI lesion loads in OCB negative patients and $O C B$ positive control patients

\begin{tabular}{lllll}
\hline & & $O C B$ positive $(n)$ & $O C B$ negative $(n)$ & P value (Wilcoxon) \\
\hline \multirow{2}{*}{ EDSS } & Mean & $5 \cdot 5(11)$ & $4.0(11)$ & \\
\multirow{2}{*}{ MR lsn load } & Median & $6 \cdot 0(11)$ & $3.5(11)$ & 0.008 \\
& Mean & $48(11)$ & $37(9)$ & 0.21 \\
& Median & $51(11)$ & $31(9)$ & 0.9
\end{tabular}

EDSS $=$ Kurtzke disability score ${ }^{15} ;$ MR lsn load = cranial MRI lesion load (see text). groups, occurring in two of $10 \mathrm{OCB}$ positive patients and one of eight OCB negative patients. The ratio of periventricular to deep white matter lesions was similar in the two patient groups. Spinal cord lesions were identified in seven of eight OCB negative patients and nine of 11 OCB positive patients with a mean spinal lesion score of 3 in both groups.

There were no significant differences in the frequencies of HLA alleles between the two groups.

\section{FOLLOW UP CSF FINDINGS IN OCB NEGATIVE} PATIENTS

Consent to follow up lumbar puncture was obtained from six of the 11 OCB negative patients included in the matched comparison, at a mean interval of four years since the initial lumbar puncture, and from the OCB negative patient in whom the study disclosed cervical cord compression. Four cases remained OCB negative by isoelectric focusing, including the patient with cervical cord compression. Three cases had become OCB positive, one of the three displaying serum bands as well as bands restricted to the CSF. The less sensitive technique of PAGE with a non-specific protein stain (Coomassie blue) failed to detect OCBs in any of the follow up samples, indicating that the response shown by isoelectric focusing was subtle. Free CSF light chains were detected by immunoblotting in two of the three cases with oligoclonal CSF.

\section{Discussion}

This study suggests three main conclusions:

(1) It confirms that the absence of OCBs in a putative case of definite multiple sclerosis should lead to a review of the clinical diagnosis and of the relevant investigations. Repeated neuroimaging and re-examination of CSF may be worthwhile in difficult cases. Nevertheless, true OCB negative clinically definite multiple sclerosis occurs, and some patients with undoubted multiple sclerosis remain persistently OCB negative over many years.

(2) The study adds to the evidence that the small group of patients with true OCB negative clinically definite multiple sclerosis has a relatively benign prognosis. Patients in the OCB negative group were significantly less disabled than the matched OCB positive patients when reassessed in this study (table 2). Retrospective scoring of disability from the clinical notes had suggested a similar conclusion (table 1).

Although we cannot be certain of the diagnosis of multiple sclerosis in the "true" OCB negative cases, these patients conformed to the Poser criteria for $\mathrm{CDMS}^{6}$ after careful review of the clinical evidence. The diagnosis was supported by the MRI appearances in the nine patients who consented to this investigation.

We matched OCB negative patients and positive controls for age, sex, disease duration, and Poser category. However, we allowed the matching of relapsing and remitting patients to secondary progressive controls and vice versa. It so happened that there were five patients 
with secondary progressive disease in the final OCB negative group and eight in the OCB positive group. A decision to avoid such pairings would have been liable to obscure any relation between OCB status and disability, the main variable under study, as the onset of significant disability is associated with the onset of the secondary progressive phase of disease. ${ }^{21}$ In other words, the hypothesis that the presence of OCBs is associated with worse disability predicted that there would be a higher proportion of patients with secondary progressive disease in the OCB positive group than among the OCB negative patients.

Examination of the pattern of scores on the Kurtzke functional systems scales $^{16}$ in OCB positive and negative cases (results not shown) does not suggest that the difference in overall disability between the two groups is explained by different patterns of functional involvement. Similar numbers in the two groups, for example, showed evidence of involvement of sensory and visual functions.

(3) The study raises the possibility that the absence of OCBs is associated with a low plaque burden. The trend towards such an association is in keeping with other reports of positive, if weak, correlations between lesion load, quantitative measures of intrathecal synthesis, and measures of disability. ${ }^{22} 23$

In conclusion, in the sample studied only $3 \%$ of patients with undoubted multiple sclerosis lack evidence of intrathecal synthesis of oligoclonal IgG. Failure to show such synthesis in a patient with suspected clinically definite multiple sclerosis should prompt a review of the clinical data, laboratory results, and neuroimaging. It may be worthwhile to repeat some or all of the investigations, as an alternative diagnosis may come to light, and a proportion of patients with OCB negative multiple sclerosis will acquire intrathecal OCBs with time. Nevertheless, some patients with undoubted multiple sclerosis remain persistently OCB negative. The present study supports the conclusion of Stendahl-Brodin and $\mathrm{Link}^{13}$ that the prognosis of true OCB negative multiple sclerosis is relatively benign. It is uncertain whether this relation between OCB status and prognosis is explained by an association with lesion load, with the intensity of inflammation, or with some other factor.
We thank Dr Alan Thompson for his helpful discussion of this work

1 Matthews WB. Laboratory diagnosis. In: McAlpine's multiple sclerosis: 2nd ed. Edinburgh: Churchill Livingstone, 1991 221.

2 Tourtellotte WW. The cerebrospinal fluid in multiple sclerosis. In: Bruyn G, ed. Handbook of clinical neurology. Vol 3 (47). Demyelinating diseases. Amsterdam: Elsevier, 1985 (47). De.
79-130.

3 Link $\mathrm{H}$. The cerebrospinal fluid in multiple sclerosis. In Swash M, Oxbury J, eds. Clinical neurology. Edinburgh: Churchill Livingstone, 1991:1128-38.

4 Reder A, Arnason B. Immunology of multiple sclerosis. In: Bruyn G, ed. Handbook of clinical neurology. Vol 3 (47). Demyelinating diseases. Amsterdam: Elsevier, 1985 : 337-95.

5 Zeman A, McLean B, Keir G, et al. The significance of serum oligoclonal bands in neurological diseases. $7 \mathrm{Neurol}$, Neurosurg Psychiatry 1993;56:32-5.

6 Poser CM, Paty DW, Scheinberg L, et al. New diagnostic criteria for multiple sclerosis: guidelines for research protocols. Ann Neurol 1983;13:227-31.

7 Miller DH, Ormerod IEC, Rudge P, et al. The early risk of multiple sclerosis following isolated acute syndromes of the brainstem and spinal cord. Ann Neurol 1989;26:635-9.

8 Sandberg-Wollheim M, Bynke H, Cronqvist S, et al. A longterm prospective study of optic neuritis: evaluation of risk factors. Ann Neurol 1990;27:386-93.

9 Sharief MK, Thompson EJ. The predictive value of intrathecal immunoglobulin synthesis and magnetic resonance imaging in acute isolated syndromes for subsequent development of multiple sclerosis. Ann Neurol 1991;29:147-51.

10 Prineas JW, Graham JS. Multiple sclerosis: zapping of surface immunoglobulin $G$ on macrophages engaged in myelin breakdown. Ann Neurol 1981;10:149-58.

11 Rudick RA, Schiffer RB, Schwetz KM, Herndon RM Multiple sclerosis: the problem of incorrect diagnosis. Arch Neurol 1986;43:578-83.

12 Farrell MA, Kaufmann JCE, Gilbert JJ, et al. Oligoclonal bands in multiple sclerosis: clinico-pathologic correlation. Neurology 1985;35:212-8.

13 Stendahl-Brodin L, Link H. Relation between benign course of multiple sclerosis and low-grade humoral immune response in cerebrospinal fluid. $f$ Neurol Neurosur Psychiatry 1980;43:102-5.

14 McDonald WI, Halliday AM. Diagnosis and classification of multiple sclerosis. Br Med Bull 1977;33:4-9.

15 Miller DH, Barkhof F, Berry I, et al. Magnetic resonance imaging in multiple sclerosis: concerted action guidelines. $\mathcal{F}$ Neurol Neurosurg Psychiatry 1991;54:683-8.

16 Kurtzke JF. Rating neurological impairment in multiple sclerosis: an expanded disability status scale. Neurology rosis: an expanded

17 Keir G, Luxton RW, Thompson EJ. Isoelectric focusing of cerebrospinal fluid IgG: an annotated update. Ann Clin Biochem 1990;27:436-43.

18 Thompson EJ. The CSF proteins: a biochemical approach Amsterdam: Elsevier, 1988:133-49.

19 Thompson AJ, Kermode AG, MacManus DG, et al. Patterns of disease activity in multiple sclerosis: a clinical and magnetic resonance imaging study. BMf 1990;300: $631-4$

20 Kidd D, Thorpe JW, Thompson AJ, et al. Spinal cord MRI using multi-array coils and fast spin echo II: findings in multiple sclerosis. Neurology 1993;43:2632-7.

21 Compston DAS. Multiple sclerosis. In: Walton J, ed. Brain's diseases of the nervous system. Oxford: Oxford University Press, 1993:373.

22 Muller FAJ, Hanny PE, Wichmann W, et al. Cerebrospinal fluid immunoglobulins and multiple sclerosis: correspondence with magnetic resonance imaging and visually evoked potential changes. Arch Neurol 1989;46:367-71.

23 Baumhefner RW, Tourtellotte WW, Syndulko K, et al. Quantitative multiple sclerosis plaque assessment with magnetic resonance imaging: its correlation with clinical parameters, evoked potentials, and intra-blood-brain-barrier IgG synthesis. Arch Neurol 1990;47:19-26. 